\title{
On the Necessity of Exogenous Variables for Load, PV and Wind Day-Ahead Forecasts using Recurrent Neural Networks
}

\author{
Henning Wilms, \\ Student Member IEEE \\ Marco Cupelli, \\ Senior Member IEEE \\ Antonello Monti, \\ Senior Member IEEE \\ Automation of Complex Power Systems Automation of Complex Power Systems Automation of Complex Power Systems \\ E.ON Energy Research Center \\ RWTH Aachen University \\ E.ON Energy Research Center \\ RWTH Aachen University \\ Aachen, Germany \\ Aachen, Germany \\ E.ON Energy Research Center \\ RWTH Aachen University \\ Aachen, Germany \\ Email: hwilms@eonerc.rwth-aachen.de Email: mcupelli@eonerc.rwth-aachen.de Email: amonti@eonerc.rwth-aachen.de
}

\begin{abstract}
In this paper we use publicly available time series data sets from the Global Energy Forecasting Competitions to evaluate the added value of exogenous, explanatory variables when forecasting wind, load and PV profiles. Two different auto-regressive models are built as well as one model that includes exogenous variables. All of models use recurrent neural networks (RNN) as their base architecture. The added value of exogenous variables is evaluated by comparing different accuracy metrics cross data set and cross model. The results show, that the autocorrelation of load and PV data sets produce reasonably good accuracies for auto-regressive predictions using RNNs, whereas wind production is far harder to forecast and the RNNs are not able to infer any suitable predictions using only a univariate time series.
\end{abstract}

\section{INTRODUCTION}

Regression as a methodology to forecast loads, wind and PV production is a relevant application for decentralized power systems with a model predictive control set up [1]. Machine Learning has recently found increased attention for regression tasks and especially time series forecasting with application in the energy sector [2], [3]. Inspired by the advances in natural language processing in handling sequences, recurrent neural networks (RNN) show various advantages and an improved performance when learning time series dependencies [4].

Time series themselves contain inherent information in their sequence of values. As such, auto-regressive forecasting exploits these dependencies in univariate time series to forecast a following value out of its previous value or values. On the other hand, time series can be enriched by providing supplementary information to each discrete time step that help describe the forecasted value in a multivariate regression.

Feature selection methods aim at reducing the complexity and computational resources when training machine learning models. The basic idea is to use only those features as input parameters that add relevant explanatory value to the model and hence improve the models overall accuracy while keeping the model as simple as possible [5]. The contribution of this paper is to evaluate how much information already lies within the time series and the sequential succession of univariate target values, itself and what is the added value in terms of increasing the forecasting accuracy of exogenous variables. To achieve this, three different RNN based forecasting architectures are built for each of the different data sets. The different architectures use exogenous variables, time stamps or simply a univariate time series for inference. To derive the added value of the exogenous variables, the forecasting accuracies of the different architecture types and data sets are compared.

In this paper we apply state of the art recurrent neural networks for the different data sets of the Global Energy Forecasting Competitions $2012^{1}$ and $2014^{1}$ (GEFCom). The findings of this paper thus show the same generality as inherent in the used data sets [6], [7].

\section{Recurrent Neural Networks And Applied MODELS}

This section gives a short introduction to recurrent neural networks and describes the models used. As naming convention, we describe the forecastable target values as labels, denoted by $Y$ and the supplementary, exogenous variables as features, denoted by $X$.

We use RNNs as base method for the purpose of evaluating the increase in prediction accuracy when feeding exogenous variables. As RNNs show promising results for capturing time series dynamics, this approach is chosen as base method over other machine learning methods [8].

\footnotetext{
${ }^{1}$ Data Sets available at http://blog.drhongtao.com/2016/07/datasets-forenergy-forecasting.html
}

(c) 2018 IEEE. Personal use of this material is permitted. 


\section{A. Recurrent Neural Networks}

Feedforward (deep) neural networks feed the inputs and activations in one forward direction to infer the label. That is, each neuron layer receives the outputs form the previous layer. The neurons of the very first layer receive the overall input values as inputs. Recurrent neural networks on the other hand possess connections to themselves. This way a neuron's output serves as its own input. These self-feeding neurons can be rolled out over time. Hence they can capture time series dynamics and include these dependencies in their predictions as previously predicted labels are considered for the current label prediction. A recurrent neuron rolled out over time is depicted in Fig. 1, in which $x$-values represent exogenous features and $y$-values represent the forecasted labels for each time step $t$.

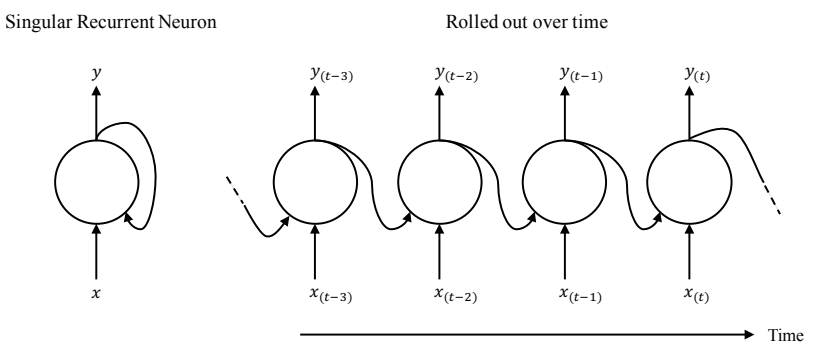

Fig. 1. recurrent neuron rolled out over time

Vanishing or exploding gradients where information gets lost during training over extensive sequences pose a challenge when training recurrent neural networks. This led to the creation of cells instead of neurons [9], [10]. These cells use state vectors as memory to store information and preserve them over time steps of arbitrary length. By storing the information in state vectors the neural network will only need to learn when to access the correct information from previous time steps for the current time step. The access to these state vectors is controlled by gate controllers that are themselves operated by neural networks. The following subsection introduces two of the most commonly used cell types.

1) LSTM Cell: Long-short-term memory cells (LSTM) in their most common form were first introduced in [11]. They possess a short term state $\mathbf{h}_{(t)}$ as well as a long term state $\mathbf{c}_{(t)}$ which together compose their memory. Short term states only exist from time step to time step. Adaptation of these states occurs by adding new and dropping old information from the long term state as well as extracting relevant information for predicting the current time step. A total of four different gates exert control over these operations. As a variant, LayerNormalizing LSTM cells (LN-LSTM) are also used in the context of this paper [12].

2) GRU Cell: Gated recurrent units (GRU) are a recently developed, simpler version of the LSTM Cells to improve training efficiency [13]. Long term and short term states are combined into one state $\mathbf{h}_{(t)}$ that subsequently require one less gate to control the state and its adaptation.

\section{B. Model Architecture and Models}

In this context, architecture refers to the underlying structure of the RNN, whereas model refers to the individually set up and trained architecture for each of the data sets.

Google's TensorFlow (TF) environment is used for implementing and training the models in python. One architecture using recurrent neural networks was built but trained in three different ways, yielding three different types of models for each data set (Fig. 2). For simplicity Fig. 2 only shows the forecast of 5 time steps, the final models are simply extended versions forecasting 24 hourly time steps.

1) Auto-regressive $R N N$ : Fig. 2a shows the applied architecture for auto-regressive forecasting (AR Model) using recurrent cells (depicted as rectangles). The architecture takes 24 previous labels as inputs to forecast the 24 following labels.

2) RNN with exogenous variables: The underlying architecture remains the same for the model that includes exogenous variables (Fig. 2b). However, instead of the previous labels, the exogenous variables are fed as inputs for each of the time steps $t$. This type of model is also known as Elman RNN [3].

3) Auto-regressive Model using Time Stamps: A third model (Elman-TS Model), fig 2c, uses the set up of the Elman model as an alternative auto-regressive model. The network here is not fed the previous labels but uses the time stamps of each time step as exogenous input variables. The time steps contain the date, i.e. year, month and day as well as hour of the day. It is here considered auto-regressive as time stamps are usually inherent to accessible time series data.

All three models can be used unidirectionally or bidirectionally. An unidirectional RNN simply forecasts one step after the other and only considers previous outputs for the current step. Bidirectional RNNs evaluate the entire time sequence and look at dependencies of future time steps with regard to the current time step as well. In a sense, they approach each time step from both directions, i.e. both ends of a time sequence [14].

\section{TRAINING, REGULARIZATION AND HYPERPARAMETERS}

This section presents how the different models were trained, what regularization techniques have been applied to prevent overfitting and which hyperparameters were adjusted to find the best possible fit for each of the models.

Individual AR, Elman-TS and Elman RNN models are trained for each of the data sets. The training process uses a gradient descent optimizer to find the model with the minimal loss, i.e. error between forecasted values and labels [14]. 
(a)

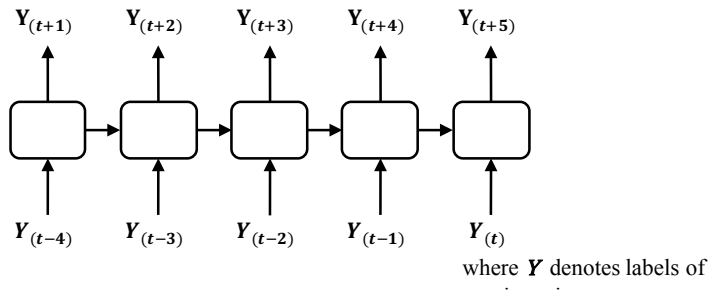

(b)

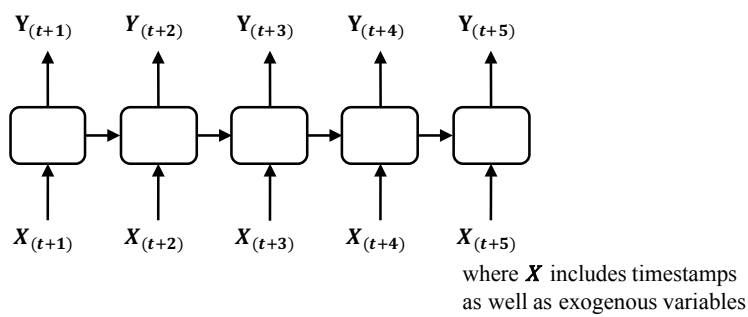

(c)

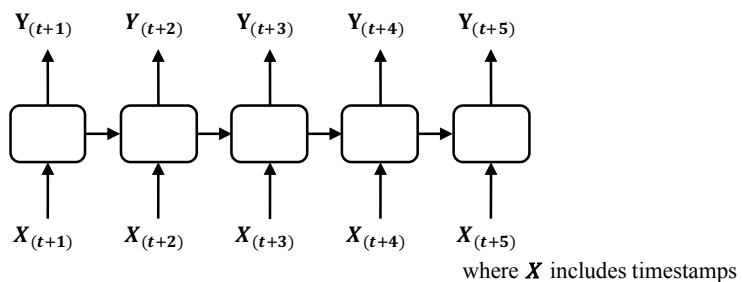

Fig. 2. Depiction of the three models

As each data set is unique, they each require individual fine tuning of the architecture and training process. These tunable parameters are called hyperparameters. Random search methodology helps to find a good combination of hyperparameters for each of the models by training a variety of models with different hyperparameter combinations and then choosing the best found model [14]. Table I shows the tunable hyperparameters as well as the search space assigned to each of the hyperparameter's optimizations.

TABLE I

TUNABLE HYPERPARAMETERS AND SEARCH SPACE FOR RANDOM SEARCH

\begin{tabular}{l|l} 
Hyperparameter & Search Space \\
\hline Cell Type & {$[$ GRU, LSTM, LN-LSTM $]$} \\
Number of neurons & {$[10,20,30,40]$} \\
Number of hidden layers & {$[1,2,3,4]$} \\
Batch size & {$[20,30,40]$} \\
Dropout keep probabilities & {$[0.6,0.7,0.8,0.9,1.0]$} \\
Bidirectional & {$[$ True, False $]$} \\
Learning rate & {$[0.001,0.01,0.1]$} \\
Gradient clip & {$[1,3,5,10]$}
\end{tabular}

The different cell types have already been introduced in section II-A. The number of neurons describes the number of hidden units of a cell and represents the size of each cell's states, i.e. the memory size. The number of hidden layers defines the number of cells stacked upon each other. Together, the number of neurons and number of layers define the size of the neural network and describe its degrees of freedom. Thus the size allows for an interpretation of how much complexity the network is capable of capturing. Bidirectionality when set to true doubles the size of the network, as the network in its specifications is rolled out twice from both ends of the time sequence.

Regularization ensures good generalization capabilities of the models and avoids overfitting. Two methods for regularization during training are applied here: dropout and early stopping. Dropout penalizes the model during training sessions, making it harder for the model to learn the dependencies of the training data set and thus forces the model to learn general dependencies. The amount of penalizing is defined by the dropout keep probability. Early stopping evaluates the generalization capabilities of the model during its training on the validation data set. Once the generalization capabilities deteriorate over several iterations and stop improving due to overfitting, it halts the training process and saves the trained model with the best generalization capabilities [14], [15].

Gradient Clipping further improves converging of the model during training by preventing a single occurrence of a high gradient to mislead the gradient descent based optimizer. Further, gradient clipping helps to solve the problem of exploding gradients [14]. The learning rate also relates to the properties of the optimizer by defining the step size by which the optimizer converges towards the minimal loss value for a training iteration. Larger learning rates lead to quicker convergence but can prevent finding the overall minimal loss [14], [15].

As it would be too computationally intensive for the optimizer to calculate the gradients on the entire data set, the data set is divided into random batches. Training then consists of several iterations where each iteration calculates the gradients using only the current batch and updating the neural networks trainable parameters (i.e. weights and biases of the neurons) following these calculated gradients. The underlying assumption for this approach is, that each random batch will include the general properties of the data set. The batch size as hyperparameter defines the size of each of these batches used per iteration [14], [15].

\section{DATA DESCRIPTION AND PREPARATION}

This section describes the data sets of the GEFCom and necessary steps for preparing the data for machine learning tasks.

\section{A. GEFCom Data Sets}

The 2012 competition provided two different data sets:

1) Load Data Set:

- Labels: Historical hourly load values of 20 zones

- Features: Temperature measurements from 11 weather stations

2) Wind Data Set:

- Labels: Normalized wind power measurements from 7 wind farms 
- Features: Meteorological wind forecasts for each of the wind farms

The 2014 competition provides four different data sets of which three are used here:

1) Load Data Set:

- Labels: Hourly load values for one zone

- Features: Supplementing temperature readings

2) Wind Data Set:

- Labels: Normalized data for 10 wind farms

- Features: Wind forecasts at 10 and $100 \mathrm{~m}$ heights

\section{3) Solar/PV Data Set:}

- Labels: Power output of 3 different PV farms

- Features: 12 supplementing features such as temperature, wind speed, air pressure, solar irradiation and cloud coverage

Some of the data sets contain missing values that need to be addressed before using the data (see section IV-B).

For all the data sets, different types of time series analysis have been performed to assess stationarity, autocorrelation and seasonality.

Augmented Dickey-Fuller Tests (ADF), KPSS Tests and Phillips-Perron Tests (PP) were used to assess stationarity. All three are statistical hypothesis test, i.e. test for acceptance or rejection of a null hypothesis. ADF and PP test the null hypothesis for an existing unit root, whereas the KPSS test contains the unit root as the alternative hypothesis. An existing unit root indicates non stationarity. To extend the ADF and PP results, KPSS tests for trend stationarity, i.e. the existence of stationarity around a deterministic trend [16], [17].

For wind, PV and load data sets they lead to the conclusions, that the considered time series possess a weak or no stationarity. Further, concluding from the results of the performed Kruskal-Wallis Tests all the time series are non-seasonal.

Fig. 3 provides an overview of the autocorrelation for each of the types of time series. For each a PV, wind and load time series, the typical autocorrelation is plotted over the first 100 lags. The autocorrelation plots show a clear pattern in the autocorrelation of the labels for the PV and the load data sets, whereas the wind time series shows a strong autocorrelation only for short lags before nearing zero. Hence, for longer lags, there is no or little explanatory value of a sequence of historic values for the current time step(s) for the wind time series, whereas a sequence of historic values will contain a strong explanatory value for the current time step(s) for the load and PV time series, as their autocorrelation pattern repeats itself and stays constant even over longer lags.

\section{B. Data Preparation}

The numerical features (e.g. temperature, wind speed) are normalized and categorical features (e.g. day, month) transformed into one-hot vectors (vectors that only contain 0 or 1 to indicate a feature characteristic) to improve learning
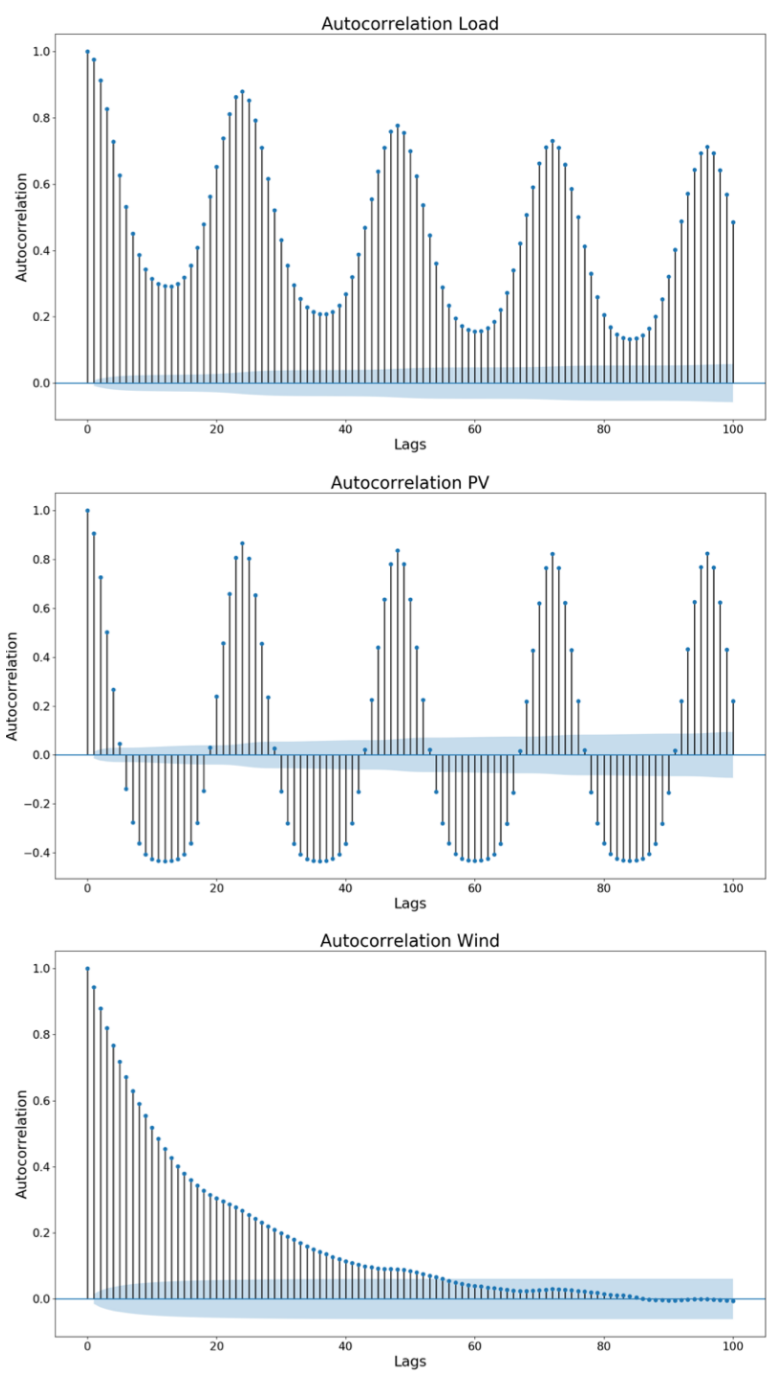

Fig. 3. Autocorrelation of three different types of time series

as well as conversion of the models. Missing values within the data sets are imputed (replace missing entries) with the median value of the entire data set for each of the features or labels [15]. Simply excluding incomplete instances from the data set is not favorable as it would break up the time series. The Wind2012 data set showed various missing features in the second half of the data set, which is why only the complete first half was used for the model training and evaluation. The Wind2014 data set was missing around $0.7 \%$ of the values that were imputed. The other data sets considered were complete.

Before training the models, each data set is split into two parts: one for training and one for valdation. This ensures, that the model's generalization capabilities are evaluated by using the second data set for calculating the model's accuracy and a possible over-fitting of the model does not unfairly benefit the model in its evaluation. 


\section{RESUlts AND Discussion}

This section provides a short overview of the used accuracy metrics used for the evaluation, presents the obtained accuracy results for each of the best found model for each of the data sets and discusses the results.

\section{A. Evaluation Parameter}

Scale dependent measures such as root mean square error (RMSE) or mean absolute error (MAE) offer good interpretability. However, their value is dependent on the scale of the underlying label and cannot be used to compare the different models across the five data sets considered here. As the aim is to evaluate the added value of exogenous variables, the performances of auto-regressive models need to be compared to non auto-regressive models cross data set. Naturally, scale independent error measures such as mean absolute percentage error (MAPE) could be used in this case. These measures however have skewed error value distributions depending on the error and label magnitude [18]. Especially as some data sets contain only normalized values, values and errors are small and these models would subsequently be adversely affected by the MAPE measure. The $R^{2}$ accuracy measure serves to evaluate and compare the models and their generalization capabilities. $R^{2}$ is called the coefficient of determination used in regression to express how well the the input parameters together with the trained model describe the variance of the output values [19]. This accuracy measure hence is independent of the scale of the forecastable labels and can be used for the goal of this paper. $R^{2}$ values lie between 0 and 1 , where a value of 1 describes a perfect explanation of the variance within the label by the model and its inputs. A value of 0 indicates that no label variance is explained. Negative values are possible when using neural networks and can be interpreted, that the predictions are in fact worse than simply predicting the mean value.

As the $R^{2}$ value offers limited numerical interpretability, it is applied here for cross model and cross data set comparison. The RMSE and MAE values are also calculated for improved interpretability.

For calculating the accuracy values of the models, the entire validation set is fed into the trained RNN and the accuracy values are calculated over the entire set of forecasts. This way, a large set of sequences is used for evaluation and some possible particularities of a single sequence on which the specific model might perform especially well, do not unfairly benefit any of the models.

\section{B. Results}

Table II shows the accuracy values for the Elman RNN, Elman-TS and AR models for each of the data sets as well as MAE and RMSE values and the underlying labels' scale for better interpretability. The presented accuracy values are the best obtained values after applying the described hyperparameter tuning using random search.
TABLE II

RESULTS

\begin{tabular}{|c|c|c|c|c|c|}
\hline Data Set & Model & $R^{2}$ & RMSE & MAE & Scale \\
\hline \multirow{3}{*}{ Wind 2012} & AR & 0.3160 & 0.2315 & 0.1827 & \multirow{3}{*}[0,1]{} \\
\hline & Elman-TS & 0.0039 & 0.2789 & 0.2292 & \\
\hline & Elman & 0.6738 & 0.1596 & 0.1188 & \\
\hline \multirow{3}{*}{ Wind 2014} & $\mathrm{AR}$ & 0.2714 & 0.2920 & 0.2415 & \multirow{3}{*}[-7.7,9.9]{} \\
\hline & Elman-TS & 0.0173 & 0.3429 & 0.3056 & \\
\hline & Elman & 0.6115 & 0.2156 & 0.1633 & \\
\hline \multirow{3}{*}{ PV2014 } & $\mathrm{AR}$ & 0.8340 & 0.1184 & 0.0695 & \multirow{3}{*}[0,1]{} \\
\hline & Elman-TS & 0.7954 & 0.1315 & 0.0805 & \\
\hline & Elman & 0.9518 & 0.0638 & 0.0357 & \\
\hline \multirow{3}{*}{ Load2012 } & $\mathrm{AR}$ & 0.7542 & 42.31 & 33.67 & \multirow{3}{*}[0,986]{} \\
\hline & Elman-TS & 0.9346 & 31.63 & 17.20 & \\
\hline & Elman & 0.9586 & 25.17 & 13.84 & \\
\hline \multirow{3}{*}{ Load2014 } & $\mathrm{AR}$ & 0.7815 & 23.32 & 17.32 & \multirow{3}{*}[16,318]{} \\
\hline & Elman-TS & 0.4866 & 35.75 & 27.97 & \\
\hline & Elman & 0.8704 & 17.96 & 12.85 & \\
\hline
\end{tabular}

\section{Conclusions and Discussion}

The results show that the Elman model that includs explanatory, exogenous variables generally outperforms the AR model as well as the Elman-TS model in terms of prediction accuracy and forecasting error on all of the presented data sets. However, the Elman-TS model shows no clear preference over the AR model or vice versa and depends on the considered data set.

For the load and PV data sets, Elman-TS and AR models reach quite satisfactory results. The label as such must therefore exhibit an underlying pattern in the succession of values that the RNN captures. As the difference between the $\mathrm{AR}$ and the Elman-TS models is small, this pattern must be extractable to the RNNs both by extrapolating previous sequences of labels as well as by mapping them to the time stamps. Load and PV profiles must therefore (1) be rather similar to its previous 24 hourly values and (2) defined by the time stamp properties year, month, day and hour to a great extent.

These assumptions are supported by analyzing the autocorrelation of the load and PV data sets. A lag of 24 shows the highest autocorrelation for load and PV. Transferring this interpretation to the influence of time stamps on the forecasting accuracy, suggests that each 24 th step on a continuous time line, i.e. hour of the day, suffices to infer good predictions. As the autocorrelation pattern is consistent, the further information provided by the time stamps might add to define the absolute value of the recurring pattern, i.e. PV always follows the same pattern, but the absolute values are higher in summer than winter months.

Forecasting wind production using the presented RNN architecture proves to be the most difficult amongst the three data types for all of the evaluated models. Even as their accuracy improves the most when adding exogenous variables in comparison to the other data sets, the resulting accuracy does not reach any satisfactory levels. The ElmanTS models did not show any generalization capabilities, 
meaning that the RNN could not learn any relevant patterns from the given time stamps. The wind production profile must therefore be mostly independent of the time stamp properties. It is however defined to some extent by the 24 previous hourly values (AR model). Nevertheless, this information alone does not explain enough variance within the label to yield high accuracy values. Both the AR and Elman-TS models show overfitting during the training sessions, irrespective of the dropout regularization. This again indicates, that the selected features do not serve well to forecast the label.

Again, these assumption are supported by analyzing the autocorrelation of the wind data sets. Over the lag of 24 steps, some autocorrelation still exists within the wind farm power outputs. This autocorrelation suffices for the AR model to build up limited generalization capabilities. However, as the autocorrelation drops towards zero over longer lags, their does not seem to be a pattern that can be mapped to the time stamp properties.

The main takeaway from the analysis is, that load and PV data sets can easily be forecasted by exploiting their autocorrelation in RNN models, even though they are both non-stationary and non-seasonal time series. Depending on the use case and its requirements for the forecast accuracy, supplementing exogenous variables can further improve the forecasting accuracy if needed. Wind however is much more difficult to forecast as there is no underlying recurring pattern and the time series values appear to be much more stochastic. Irrespective of the use case for the use of wind forecasts, exogenous variables are needed to produce reasonable good RNN based forecasting models. Furthermore, as the Elman models for the wind forecasts did not reach high accuracy values, further data preparation such as de-trending or de-noising could enhance the forecasts. Also, looking at this forecasting challenge from a domain specific perspective, i.e. a physics based approach or a hybrid model combining physics and statistical modeling, could also provide useful insights to improve the accuracy, rather than simply applying the best practice machine learning approach. We expect that the need of exogenous variables for wind forecasting is the same when using other forecasting techniques.

\section{Further Research}

The findings of this paper should be reassessed using different wind, load and PV data sets and different aggregation levels than the ones used here to evaluate whether the forecasting accuracies are similar. This will provide a better judgment on the generality of the presented findings.

\section{REFERENCES}

[1] C. A. Hans, P. Sopasakis, A. Bemporad, J. Raisch, and C. ReinckeCollon, "Scenario-based model predictive operation control of islanded microgrids," in Decision and Control (CDC), 2015 IEEE 54th Annual Conference on. IEEE, Conference Proceedings, pp. 3272-3277.
[2] Z. Jian, X. Cencen, Z. Ziang, and L. Xiaohua, "Electric load forecasting in smart grids using long-short-term-memory based recurrent neural network," in 2017 51st Annual Conference on Information Sciences and Systems (CISS), Conference Proceedings, pp. 1-6.

[3] F. M. Bianchi, E. Maiorino, M. C. Kampffmeyer, A. Rizzi, and R. Jenssen, "An overview and comparative analysis of recurrent neural networks for short term load forecasting," arXiv preprint arXiv:1705.04378, 2017.

[4] Z. C. Lipton, J. Berkowitz, and C. Elkan, "A critical review of recurrent neural networks for sequence learning," arXiv preprint arXiv:1506.00019, 2015.

[5] G. Chandrashekar and F. Sahin, "A survey on feature selection methods," Computers and Electrical Engineering, vol. 40, no. 1, pp. 16-28, 2014.

[6] T. Hong, P. Pinson, and S. Fan, "Global energy forecasting competition 2012," International Journal of Forecasting, vol. 30, no. 2, pp. 357363, 2014.

[7] T. Hong, P. Pinson, S. Fan, H. Zareipour, A. Troccoli, and R. J. Hyndman, "Probabilistic energy forecasting: Global energy forecasting competition 2014 and beyond," International Journal of Forecasting, vol. 32, no. 3, pp. 896-913, 2016.

[8] J. T. Connor, R. D. Martin, and L. E. Atlas, "Recurrent neural networks and robust time series prediction," IEEE Transactions on Neural Networks, vol. 5, no. 2, pp. 240-254, 1994.

[9] S. Hochreiter, "The vanishing gradient problem during learning recurrent neural nets and problem solutions," International Journal of Uncertainty, Fuzziness and Knowledge-Based Systems, vol. 06, no. 02, pp. 107-116, 1998. [Online]. Available: https://doi.org/10.1142/S0218488598000094

[10] Y. Bengio, P. Simard, and P. Frasconi, "Learning long-term dependencies with gradient descent is difficult," IEEE transactions on neural networks, vol. 5, no. 2, pp. 157-166, 1994.

[11] F. A. Gers, J. Schmidhuber, and F. Cummins, "Learning to forget: continual prediction with lstm," in 1999 Ninth International Conference on Artificial Neural Networks ICANN 99. (Conf. Publ. No. 470), vol. 2, Conference Proceedings, pp. 850-855 vol.2.

[12] J. Lei Ba, J. R. Kiros, and G. E. Hinton, "Layer normalization," ArXiv e-prints, vol. 1607, p. arXiv:1607.06450, 2016. [Online]. Available: http://adsabs.harvard.edu/abs/2016arXiv160706450L

[13] K. Cho, B. van Merrienboer, C. Gulcehre, D. Bahdanau, F. Bougares, H. Schwenk, and Y. Bengio, "Learning phrase representations using rnn encoder-decoder for statistical machine translation," ArXiv e-prints, vol. 1406, p. arXiv:1406.1078, 2014. [Online]. Available: http://adsabs.harvard.edu/abs/2014arXiv1406.1078C

[14] I. Goodfellow, Y. Bengio, and A. Courville, Deep learning, ser. Adaptive computation and machine learning. Cambridge, Massachusetts: The MIT Press, 2016.

[15] A. Geron, Hands-On Machine Learning with Scikit-Learn and TensorFlow: Concepts, Tools, and Techniques to Build Intelligent Systems. O'Reilly Media, 2017.

[16] J. S. Armstrong, Principles of forecasting: a handbook for researchers and practitioners. Springer Science and Business Media, 2001, vol. 30 .

[17] D. Kwiatkowski, P. C. Phillips, P. Schmidt, and Y. Shin, "Testing the null hypothesis of stationarity against the alternative of a unit root: How sure are we that economic time series have a unit root?" Journal of econometrics, vol. 54, no. 1-3, pp. 159-178, 1992.

[18] R. J. Hyndman and A. B. Koehler, "Another look at measures of forecast accuracy," International journal of forecasting, vol. 22, no. 4, pp. 679-688, 2006.

[19] I. Guyon and A. Elisseeff, "An introduction to variable and feature selection," Journal of machine learning research, vol. 3, no. Mar, pp. 1157-1182, 2003. 